\title{
Influence of the oxygen microenvironment on the proangiogenic potential of human endothelial colony forming cells
}

\author{
Martin L. Decaris · Chang I. Lee · Mervin C. Yoder • \\ Alice F. Tarantal $\cdot$ J. Kent Leach
}

Received: 4 April 2009/Accepted: 9 June 2009/Published online: 21 June 2009

(c) The Author(s) 2009. This article is published with open access at Springerlink.com

\begin{abstract}
Therapeutic angiogenesis is a promising strategy to promote the formation of new or collateral vessels for tissue regeneration and repair. Since changes in tissue oxygen concentrations are known to stimulate numerous cell functions, these studies have focused on the oxygen microenvironment and its role on the angiogenic potential of endothelial cells. We analyzed the proangiogenic potential of human endothelial colony-forming cells (hECFCs), a highly proliferative population of circulating endothelial progenitor cells, and compared outcomes to human dermal microvascular cells (HMVECs) under oxygen tensions ranging from $1 \%$ to $21 \% \mathrm{O}_{2}$, representative of ischemic or healthy tissues and standard culture conditions. Compared to HMVECs, hECFCs (1) exhibited significantly greater proliferation in both ischemic conditions and ambient air; (2) demonstrated increased migration compared to HMVECs
\end{abstract}

M. L. Decaris · J. K. Leach $(\square)$

Department of Biomedical Engineering, University

of California, Davis, 451 Health Sciences Drive,

Davis, CA 95616, USA

e-mail: jkleach@ucdavis.edu

C. I. Lee · M. C. Yoder - A. F. Tarantal

Center of Excellence in Translational Human Stem Cell

Research, University of California, Davis, Davis, CA, USA

C. I. Lee · A. F. Tarantal

California National Primate Research Center, University

of California, Davis, Davis, CA, USA

C. I. Lee · A. F. Tarantal

Departments of Pediatrics and Cell Biology and Human

Anatomy, University of California, Davis, Davis, CA, USA

M. C. Yoder

Wells Center for Pediatric Research, Department of Pediatrics, Indiana University School of Medicine, Indianapolis, IN, USA when exposed to chemotactic gradients in reduced oxygen; and (3) exhibited comparable or superior proangiogenic potential in reduced oxygen conditions when assessed using a vessel-forming assay. These data demonstrate that the angiogenic potential of both endothelial populations is influenced by the local oxygen microenvironment. However, hECFCs exhibit a robust angiogenic potential in oxygen conditions representative of physiologic, ischemic, or ambient air conditions, and these findings suggest that hECFCs may be a superior cell source for use in cell-based approaches for the neovascularization of ischemic or engineered tissues.

Keywords Angiogenesis · Endothelial cells · Endothelial progenitor cells $\cdot$ Neovascularization

\section{Introduction}

Cardiovascular diseases caused by atherosclerotic narrowing or occlusion of arteries remain the leading cause of morbidity and mortality in developed countries [1]. Although percutaneous revascularization and bypass surgery provide a means to restore blood flow to ischemic tissues, not all patients with symptomatic ischemic diseases are suitable candidates for these interventions. Therapeutic angiogenesis is a promising treatment option for these patients. A number of new potential therapies are under investigation to revitalize ischemic tissues including the delivery of recombinant angiogenic growth factors and cytokines, the use of gene therapy to deliver plasmid DNA encoding for proangiogenic factors, and the implantation of proangiogenic biomaterials [2-4]. Despite promising results, these therapies suffer from limitations including the dependence of host cells to respond to these inductive 
cues, reliance upon the kinetics and duration of protein presentation for efficacy, and the potential inability of donor cells to adjust to the hypoxic environment of the damaged tissue. Cell-based approaches to therapeutic angiogenesis may provide a viable alternative for improved neovascularization by transplanting cells that directly participate or indirectly promote the formation of new vessels.

Therapeutic angiogenesis may be achieved through a cell-based approach. Compared to pharmacological strategies $[5,6]$, the transplant of cells of the endothelial lineage can accelerate this process by formation of new capillaries. Local implantation of terminally differentiated endothelial cells, both microvascular such as human dermal microvascular cells (HMVECs) and umbilical vein-derived endothelial cells (HUVECs), has yielded functional vessels in rodent models, but these neovessels only survive for a short period of time [7-12]. Alternatively, endothelial progenitor cells (EPCs) represent a source of endothelial cells that may be isolated from adult peripheral or umbilical cord blood and possess high proliferative potential; thus, eliminating the challenges in expanding autologous HMVECs for graft endothelialization or transplantation [13-17]. Endothelial colony-forming cells (ECFCs) represent the only EPCs that display clonal proliferative potential, high levels of telomerase, form de novo vessels upon implantation in vivo, and spontaneously anastomose with the host vasculature [18]. However, the angiogenic properties of ECFCs in ischemic or physiologic oxygen microenvironments remain to be fully explored.

The oxygen microenvironment is a potent stimulator of cell behavior. Oxygen tension throughout the body ranges from nearly anoxic in cartilage and ischemic cardiac or cerebral tissues to approximately $13 \%$ in arteries [19]. Microvascular endothelial cells from various sources exhibit significant differences in their proliferative, migratory, and tubule/vessel-forming capacity as a result of changes in environmental oxygen tension [20-22]. Endothelial cells may also increase the production of proangiogenic growth factors including vascular endothelial growth factor (VEGF) and collagenases such as MMP-9 in response to hypoxic conditions [23-25], each of which play an important role in the process of cell-mediated vessel formation.

We hypothesized that local oxygen tension would affect the vessel-forming behavior of cells of the endothelial lineage, and that endothelial cells from different sources may exhibit different responses to hypoxic conditions. To explore this hypothesis, we compared the proangiogenic potential of human ECFCs (hECFCs) to HMVECs in controlled oxygen tensions in vitro and demonstrated that hECFCs possess comparable or enhanced proangiogenic potential at all oxygen tensions studied when compared to HMVECs. Thus, hECFCs are likely a superior cell source for use in cell-based approaches focused on the neovascularization of ischemic or engineered tissues.

\section{Materials and methods}

\section{Cell culture}

Human umbilical cord blood ECFCs were isolated using a protocol approved by the Institutional Review Board of the Indiana University School of Medicine as previously described [18]. The adherent ECFCs were cultured in T-75 culture flasks (Nunc) coated with a layer of $5 \mu \mathrm{g} / \mathrm{cm}^{2}$ rat tail collagen I (BD Biosciences) in $\mathrm{EGM}^{\circledR}-2$ media supplemented with Lonza's SingleQuot supplement (hydrocortisone, gentamycin, human VEGF, human fibroblast growth factor (FGF), human epidermal growth factor (EGF), human insulin-like growth factor (IGF), and heparin) and supplemented with $10 \%$ fetal bovine serum (FBS, JR Scientific), $1 \%$ penicillin/streptomycin, and $0.1 \%$ amphotericin $\beta$ (Mediatech). Adult dermal HMVECs were received in cryogenic ampoules (Clonetics ${ }^{\circledR}$ HMVEC-dAd-Adult Human Dermal Microvascular Endothelial Cells, Lonza) and cultured in EGM $^{\circledR}-2$ MV Microvascular Endothelial Cell Growth Medium-2 supplemented with the Bulletkit supplement $(5 \%$ FBS, hydrocortisone, gentamycin, human VEGF, human FGF, human EGF, human IGF, and ascorbic acid). Both hECFCs and HMVECs were maintained in standard $\mathrm{CO}_{2}$ incubators at $37^{\circ} \mathrm{C}$ prior to experimentation.

\section{Controlling the oxygen microenvironment}

The oxygen microenvironment was manipulated using commercially available airtight chambers (Billups-Rothenberg). A known mixture of $\mathrm{CO}_{2}$ and $\mathrm{N}_{2}$ was pumped into the chamber and the $\mathrm{O}_{2}$ levels inside the chamber were monitored with an oxygen sensor (Billups-Rothenberg) attached to the outlet tube. Once the desired oxygen level was reached, the chamber was sealed, leaving an interior atmosphere of $5 \% \mathrm{CO}_{2}$ and a precise mix of $\mathrm{O}_{2}$ and $\mathrm{N}_{2}$. A small tray of water was placed in the hypoxia chambers to maintain humidity after sealing. Sealed chambers were placed in a $37^{\circ} \mathrm{C}$ incubator during experiments. Experimental endpoints, assays, and oxygen tensions employed are described in Table 1.

\section{Cellular proliferation}

The role of the oxygen microenvironment on hECFCs and HMVECs mitogenic potential was assessed by quantitating cellular proliferation when cultured in varied oxygen conditions ( $\ll 1 \%$ representing near anoxic conditions, $4,8,12$, 16, and $21 \% \mathrm{O}_{2}$ ). hECFCs and HMVECs were seeded in 
Table 1 Experimental endpoints, assays, and oxygen tensions employed

\begin{tabular}{llr}
\hline Endpoint & Assay & Oxygen tension applied (\%) \\
\hline Cellular growth & EC proliferation & $\ll 1,4,8,12,16$, and 21 \\
Migration & Boyden chamber assay & 1,5, and 21 \\
Tubulogenesis & Matrigel tubule formation assay & 1,5, and 21 \\
Apoptosis & Caspase 3/7 activity & 1,5, and 21 \\
LDL uptake & Acetylated LDL/fluorescence & 1,5 , and 21 \\
Oxygen responsiveness & Western blotting for HIF-1 $\alpha$ & 1,5, and 21 \\
\hline
\end{tabular}

$E C$ endothelial cells, $L D L$ low density lipoprotein, $H I F-1 \alpha$ hypoxia inducible factor 1-alpha, $V E G F$ vascular endothelial growth factor

complete media $\left(7,500\right.$ cells $/ \mathrm{cm}^{2}$ in EGM-2MV with $5 \%$ FBS) in 6-well culture dishes and allowed to attach overnight. To initially determine the optimal growth substrate for each population, hECFCs and HMVECs were seeded on wells coated with $5 \mu \mathrm{g} / \mathrm{cm}^{2}$ rat tail collagen or on tissue culture plastic. Subsequent experiments were performed with hECFCs grown on collagen and HMVECs grown on plastic. After attachment, media was changed and plates were placed in the desired oxygen tension for $72 \mathrm{~h}$. Each well was rinsed with Hank's Balanced Salt Solution (Gibco) and cells were trypsinized and scraped from the well surfaces prior to counting. Cell counts of each well were performed in triplicate with a Z1 Dual Coulter Counter (Beckman).

\section{Migration}

Human endothelial colony-forming cells and HMVEC migration at various oxygen tensions were evaluated using a modified Boyden chamber assay as previously described [26]. Briefly, 24-well fluoroblok transwells $(3 \mu \mathrm{m}$ pore size, BD Biosciences) were coated with a thin layer of gelatin solution $(0.1 \%$ bovine skin gelatin, Sigma). Cells $\left(1 \times 10^{5}\right.$ cells/well $)$ were seeded on the top of the transwells in $300 \mu \mathrm{L}$ serum-free growth factor deficient EGM2MV supplemented with $0.1 \%$ fatty acid-free BSA (Sigma). Transwells were then placed over complete EGM2 containing $10 \%$ FBS to create a positive chemotactic gradient, while serum-free media served as the negative control. Plates were then placed in hypoxia chambers or standard $\mathrm{CO}_{2}$ incubators for $24 \mathrm{~h}$. Cells that migrated to the bottom of the transwell were stained via incubation with calcein AM ( $3 \mu \mathrm{g} / \mathrm{mL}$ in HBSS, Anaspec) for $30 \mathrm{~min}$, and fluorescence was quantitated using a microplate reader (Synergy HTTR) at 485/530 $\mathrm{nm}$.

\section{Tubule formation}

The tubulogenic capacity of each cell type was determined when cultured in varying oxygen conditions. Briefly,
Matrigel (150 $\mu \mathrm{L}, \mathrm{BD}$ Biosciences) was pipetted into 24well plates and allowed to gel at $37^{\circ} \mathrm{C}$ for $1 \mathrm{~h}$. Cells were seeded on Matrigel at $2 \times 10^{4}$ cells $/ \mathrm{cm}^{2}$ in $500 \mu \mathrm{L}$ of complete EGM-2MV supplemented with 5\% FBS. Cells were placed in the appropriate oxygen microenvironment as described above, cultured for $6 \mathrm{~h}$, and images of tubule formation were captured using a Nikon Eclipse TE2000-U and SpotRT digital camera.

Acetylated LDL uptake

Acetylated low density lipoprotein labeled with $1,1^{\prime}$-dioctadecyl-3,3,3', $3^{\prime}$-tetramethylindo-carbocyanine perchlorate (DiI-Ac-LDL) (Biomedical Technologies) was used to characterize ac-LDL uptake by hECFCs and HMVECs at varying oxygen tensions. Cells were seeded in 6-well plates at 7,500 cells $/ \mathrm{cm}^{2}$ as described above and placed in 1,5 , or $21 \%$ oxygen for $72 \mathrm{~h}$. Cells were then incubated for $4 \mathrm{~h}$ in complete EGM-2MV + 5\% FBS containing $10 \mu \mathrm{g} / \mathrm{mL}$ of DiI-Ac-LDL at their respective oxygen tensions followed by multiple media rinses. Images of Ac-LDL uptake were captured by fluorescence microscopy using a Nikon Eclipse TE2000-U.

\section{Apoptosis}

The apoptotic response of hECFCs and HMVECs to different oxygen conditions was determined by quantitating caspase 3/7 activity (Caspase-Glo 3/7 Assay, Promega). Cells were seeded $\left(5 \times 10^{3}\right.$ cells/well) on Nunclon Surface 96well white cell culture plates (Nunc) in $100 \mu \mathrm{L}$ complete EGM-2MV containing 5\% FBS. After $24 \mathrm{~h}$, nonadherent cells were rinsed away with PBS, media was replaced, and the 96-well plates were placed in hypoxia chambers or standard $\mathrm{CO}_{2}$ incubators at $37^{\circ} \mathrm{C}$ for an additional $24 \mathrm{~h}$. Plates were then equilibrated to room temperature and $100 \mu \mathrm{L}$ of the luminescent caspase substrate/buffer mix was added to each well. Plates were agitated at $400 \mathrm{rpm}$ for $30 \mathrm{~s}$ and allowed to sit for $60 \mathrm{~min}$. Luminescence of each well was quantitated using a microplate reader. Additional wells 
of cells were also seeded in order to normalize the caspase 3/ 7 quantities to cell number. $100 \mu \mathrm{L}$ of passive lysis buffer (Promega) was applied to each additional well at the conclusion of the assay and cell DNA content was determined following the manufacturer's instructions (Quant-iT PicoGreen dsDNA Assay Kit, Invitrogen).

\section{Western blot analysis}

Human endothelial colony-forming cells and HMVECs were seeded on 6 well plates $\left(5 \times 10^{4}\right.$ cells $\left./ \mathrm{cm}^{2}\right)$ and allowed to attach overnight. The plates were then placed in hypoxia chambers, standard cell culture incubators, or cultured in ambient air with or without the presence of the Hypoxia Inducible Factor 1-alpha (HIF-1 $\alpha)$ stabilizer $\mathrm{CoCl}_{2}(100 \mu \mathrm{m}$, Sigma) for $4 \mathrm{~h}$, removed and rinsed with PBS, followed by total protein collection in $4 \mathrm{x}$ sample buffer $(20 \%$ glycerol, $4 \%$ SDS, $0.05 \%$ bromophenol blue, $160 \mathrm{mM}$ Tris- $\mathrm{HCl}$, and $200 \mathrm{mM}$ DTT). Protein concentration was determined using the Amido Black method, a solid-phase method for the quantitation of protein in the presence of sodium dodecyl sulfate and other interfering substances [27]. 20-40 $\mu \mathrm{g}$ of protein per sample were resolved in $4-20 \%$ Tris- $\mathrm{HCl}$ acrylamide gels and transferred onto $0.2 \mathrm{~mm}$ nitrocellulose. Blots were blocked in $0.5 \%$ nonfat milk in Tris-buffered saline with $0.05 \%$ Tween-20 (TBST) for $2 \mathrm{~h}$ and probed with rabbit anti-human polyclonal antibody to HIF- $1 \alpha(1: 400$ in blocking buffer; Cayman Chemical, 10006421) overnight at $4^{\circ} \mathrm{C}$. Membranes were washed and probed with horseradish peroxidase-conjugated secondary antibodies at 1:2,500 (Cayman) and reactive bands were visualized using enhanced chemiluminescence and X-ray film. Membranes were then stripped and blocked a second time with 5\% nonfat milk in TBST for $1 \mathrm{~h}$ and probed with $\alpha$-tubulin (11H10) Rabbit monoclonal antibody (1:1,000 in blocking buffer; Cell Signaling, 2125S) overnight at $4^{\circ} \mathrm{C}$. Secondary antibodies and visualization were carried out as previously stated.

\section{Statistical analysis}

The values on the graphs represent means and standard error unless otherwise stated. Statistical analysis was performed using a paired Student's $t$-test. $P$ values less than 0.05 were considered statistically significant.

\section{Results}

Cellular proliferation

We initially examined the proliferative capacity of hECFCs and HMVECs in ambient air both in the presence and absence of rat tail collagen I on the culture plates, as previous reports described ECFC culture on this extracellular matrix protein [28]. hECFC proliferation was significantly increased by the presence of the collagen substrate, while HMVEC proliferation was significantly decreased (Fig. 1a). Therefore, we elected to culture each cell population on the surface that promoted optimal proliferation for each respective cell type for the remainder of the studies.

Compared to HMVECs, hECFCs exhibited increased proliferation at both low $(\ll 1 \%, 4 \%)$ and high oxygen tensions $(16,21 \%)$ (Fig. 1b). No significant differences in proliferation were noted between hECFCs and HMVECs at mid-range oxygen levels $(8,12 \%)$. hECFCs appeared to
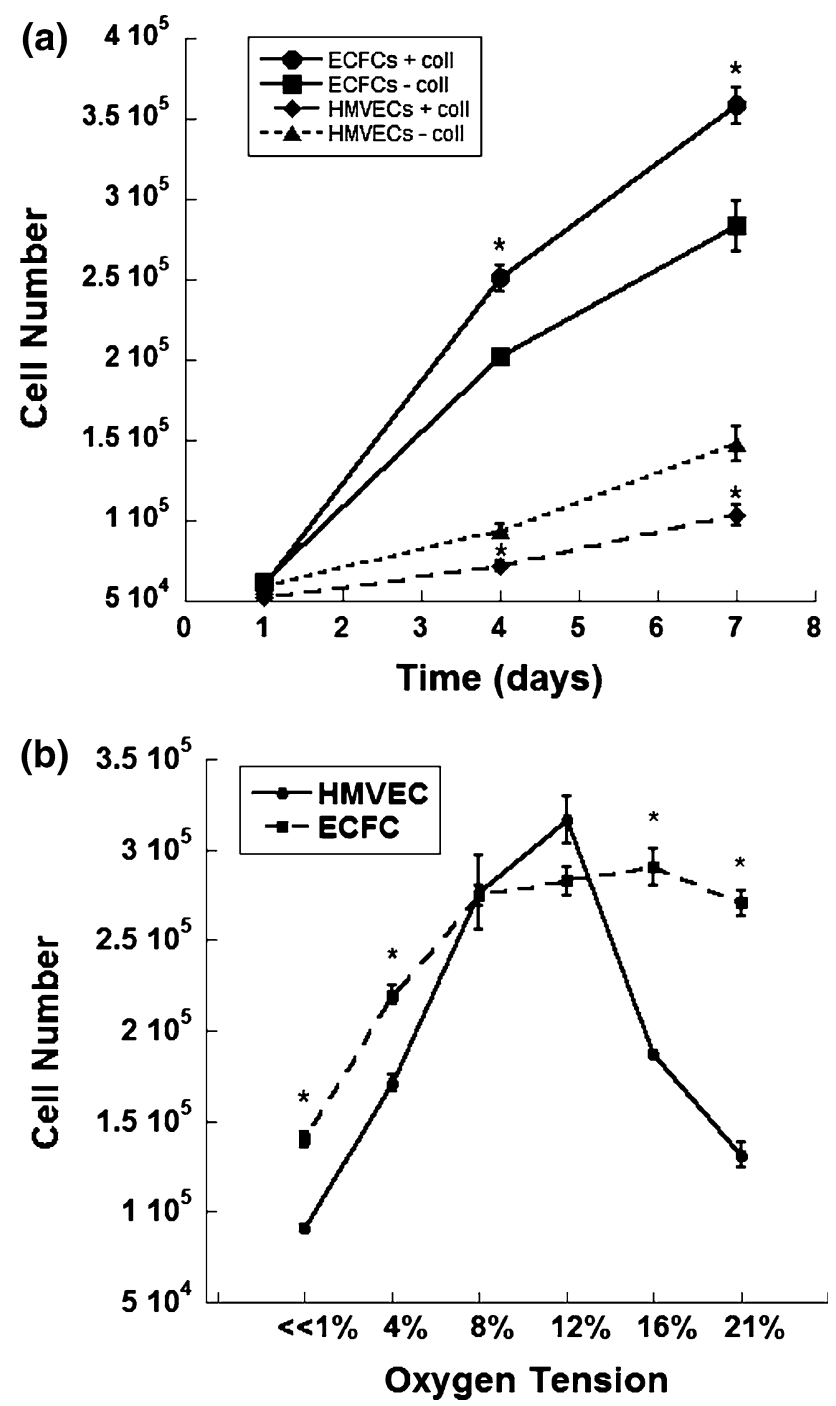

Fig. 1 a hECFC and HMVEC proliferation on collagen I. $* P<0.05$ versus same cell type cultured in the absence of collagen. Data are mean \pm SE $(n=3)$. b Proliferation of hECFCs and HMVECs at varying oxygen tensions. $* P<0.001$ versus HMVECs at corresponding oxygen tension. Data are mean $\pm \operatorname{SE}(n=6)$ 
Table 2 Population doubling times of hECFC and HMVEC cultured in varied oxygen tensions

\begin{tabular}{lll}
\hline $\begin{array}{l}\text { Oxygen } \\
\text { tension }(\%)\end{array}$ & $\begin{array}{l}\text { hECFC } \\
\text { doubling } \\
\text { time (days) }\end{array}$ & $\begin{array}{l}\text { HMVEC } \\
\text { doubling } \\
\text { time (days) }\end{array}$ \\
\hline$\ll 1$ & $3.12 \pm 0.15^{*}$ & $9.00 \pm 0.81$ \\
4 & $1.85 \pm 0.03^{*}$ & $2.40 \pm 0.07$ \\
8 & $1.54 \pm 0.02$ & $1.57 \pm 0.08$ \\
12 & $1.51 \pm 0.03$ & $1.40 \pm 0.04$ \\
16 & $1.40 \pm 0.04^{*}$ & $2.15 \pm 0.02$ \\
21 & $1.56 \pm 0.03^{*}$ & $3.58 \pm 0.31$ \\
\hline
\end{tabular}

$* P<0.05$ versus HMVECs at same $\mathrm{O}_{2}$ tension

show a proliferation plateau at mid to high oxygen tensions, while HMVECs peaked at mid-range oxygen tensions and returned to a lower proliferative capacity at higher oxygen tensions. Doubling times were calculated for both cell types at each oxygen tension using the following equation: $N=N_{0} \times \exp \left(k_{\mathrm{p}} \times t\right)$, where $N$ represents the final cell number, $N_{0}$ is the initial cell number, $k_{\mathrm{p}}$ is the proliferation rate constant, and t represents the culture time (Table 2). The greatest differences in cell proliferation doubling times were observed between hECFCs and HMVECs at $\ll 1 \% \mathrm{O}_{2}$ (3.12 versus 9.00 days, respectively) and $21 \% \mathrm{O}_{2}$ (1.56 versus 3.58 days, respectively).

\section{Cell migration}

Cell migration assays performed at three distinct oxygen tensions $\left(1,5\right.$, and $\left.21 \% \mathrm{O}_{2}\right)$ revealed a statistically significant increase in the chemotactic response of hECFCs versus HMVECs in each condition (Fig. 2). Compared to no gradient at 1,5 , or $21 \% \mathrm{O}_{2}$, hECFC migration increased 9.69-, 7.86-, and 19.67-fold, while HMVEC migration increased 3.56-, 4.35-, and 5.68-fold $(P<0.05$ for each

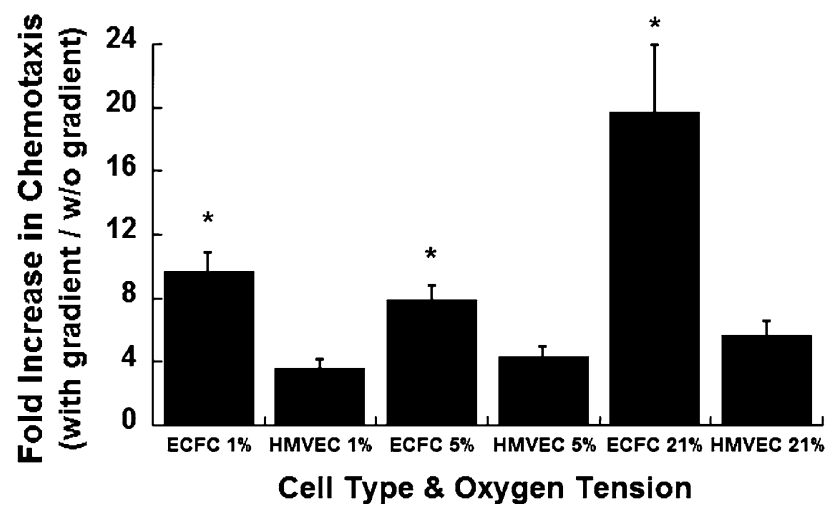

Fig. 2 Fold increase in cell migration when exposed to a chemotactic gradient at varying oxygen tensions. Data are mean $\pm \mathrm{SE}(n=4)$. $* P<0.05$ versus HMVECs at same $\mathrm{O}_{2}$ tension oxygen tension). Cell migration was visibly confirmed by imaging the bottom of transwells stained with calcein using inverted fluorescence microscopy (Fig. 3). A comparison of calcein uptake and fluorescence from an identical number of hECFCs and HMVECs revealed no significant differences (data not shown).

Tubule formation

Morphological differences were clearly observed for tubules generated by both hECFCs and HMVECs at differing oxygen tensions. Specifically, both hECFCs and HMVECs cultured in 1 and $5 \% \mathrm{O}_{2}$ produced well-rounded, circular tubules with borders typically comprised of more than one cell (Fig. 4a, b, d, e). Tubules formed at $21 \% \mathrm{O}_{2}$ by both cell populations were more polygonal in shape, less likely to be fully enclosed, and typically bordered by a layer of only one cell (Fig. 4c, f). Regardless of cell type, individual cell morphology appeared to adjust to the oxygen tension, with cells in reduced oxygen environments maintaining their cobblestone-like appearance, and those in higher oxygen conditions exhibiting an elongated morphology. Compared to hECFCs, HMVECs were less likely to adhere and remain viable at each oxygen tension when seeded on Matrigel as demonstrated by numerous floating cells (Fig. 4d-f).

\section{Apoptosis}

We detected differences in markers of programmed cell death between HMVECs and hECFCs cultured at 1, 5, or $21 \%$ oxygen over $24 \mathrm{~h}$. hECFCs expressed significantly higher caspase activity than HMVECs when cultured at $21 \% \mathrm{O}_{2}$ (Fig. 5). In addition, both hECFCs and HMVECs exhibited significant increases in caspase activity when cultured at $1 \% \mathrm{O}_{2}$ compared to $5 \%$ and $21 \% \mathrm{O}_{2}$, and these levels were statistically similar. Markers of apoptosis were statistically similar for each individual cell type when cultured in $5 \% \mathrm{O}_{2}$ and ambient air.

Acetylated LDL uptake

The ability to engulf acetylated LDL is a characteristic trait of cells of the endothelial phenotype, and this property is commonly used to isolate and identify endothelial cells from other cell types and monitor cellular metabolism [29]. Acetylated LDL incorporation assays performed at three distinct oxygen tensions $\left(1,5\right.$, and $\left.21 \% \mathrm{O}_{2}\right)$ revealed no qualitative differences between hECFCs and HMVECs (Fig. 6). Upon quantitating the number of cells per field of view with internalized fluorescence, we observed that all 
Fig. 3 Fluorescence microscopy images of transwell undersides reveal calceinstained hECFCs $(\mathbf{a}, \mathbf{b})$ and HMVECs (c, d) that crossed the barrier with $(\mathbf{a}, \mathbf{c})$ or without $(\mathbf{b}$, d) a chemotactic gradient in $1 \%$ oxygen. Images are representative of four independent experiments. Scale bars represent $200 \mu \mathrm{m}$
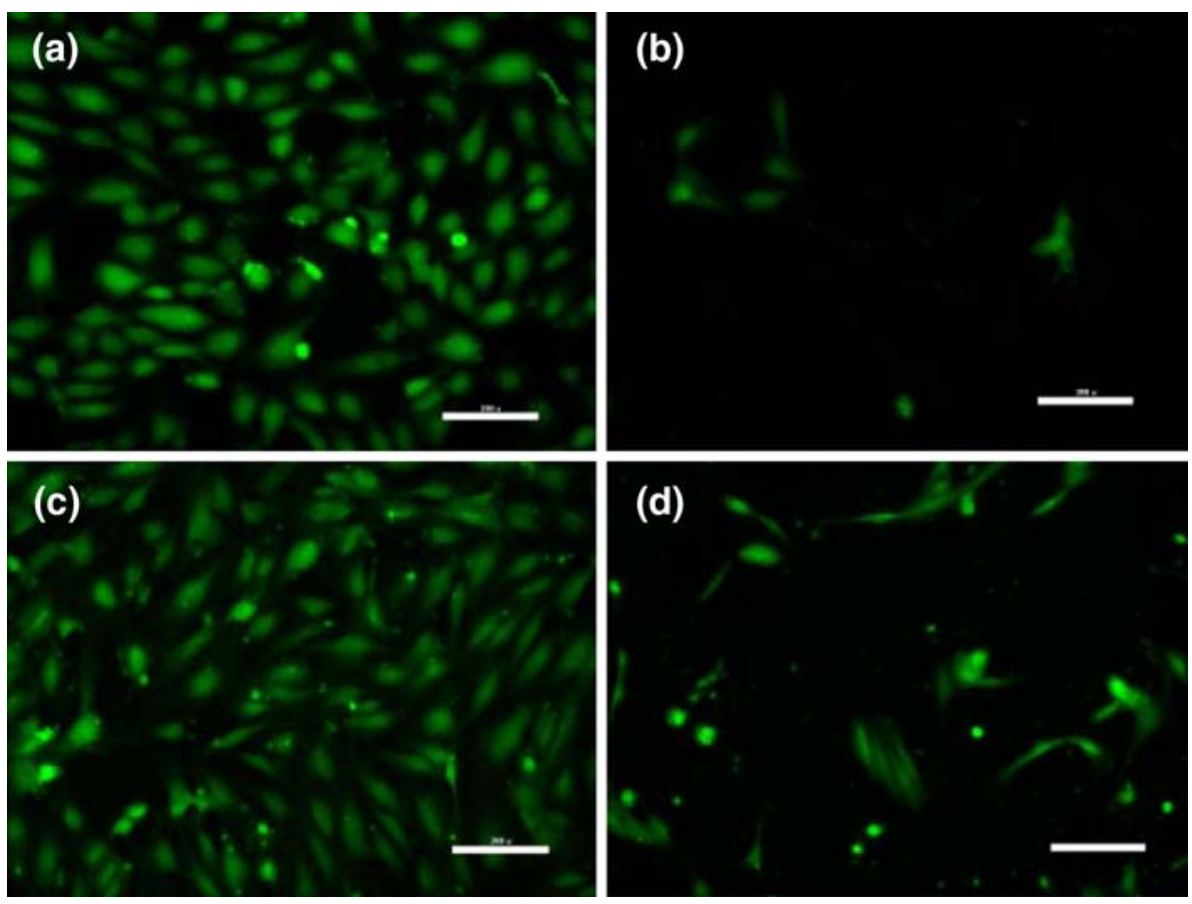

Fig. 4 Tubulogenesis with hECFCs (a-c) and HMVECs (d-f) cultured at 1,5 , or $21 \%$ oxygen. Images are representative of four independent experiments. Scale bars represent $200 \mu \mathrm{m}$

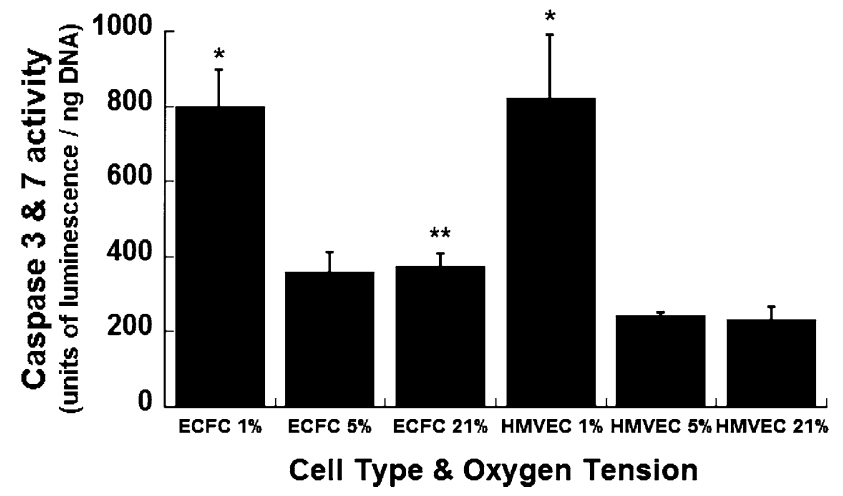

Fig. 5 Apoptotic response of hECFCs and HMVECs under varying oxygen tensions. ${ }^{*} P<0.05$ versus same cell type at 5 or $21 \% \mathrm{O}_{2}$. Data are mean $\pm \mathrm{SE}(n=4)$. $* * P<0.05$ versus HMVECs at $21 \% \mathrm{O}_{2}$

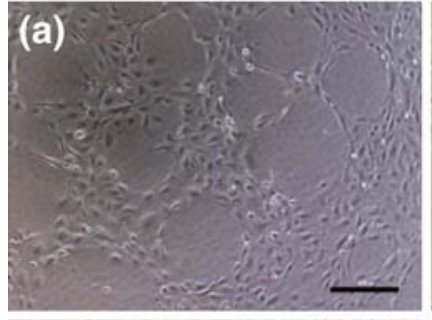

$5 \%$
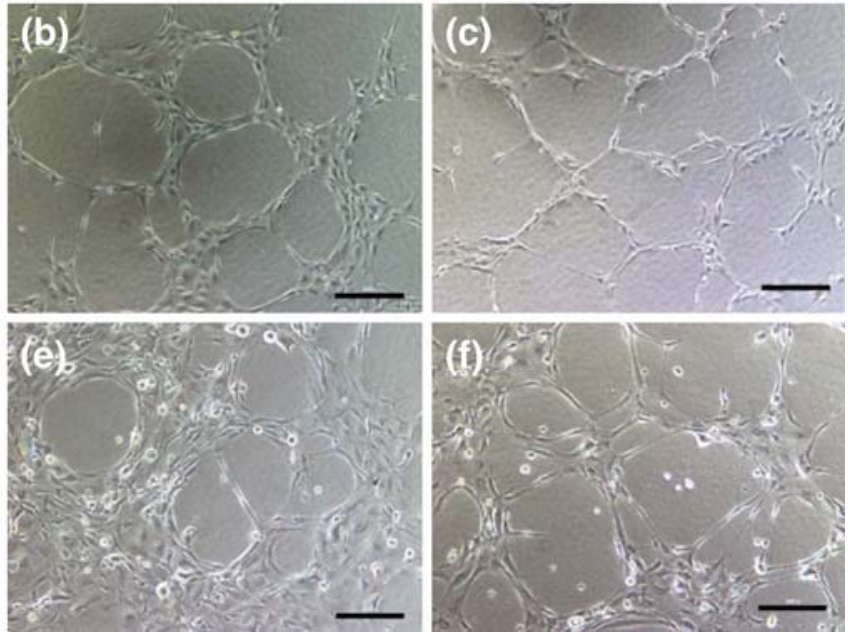

cells, regardless of oxygen tension, exhibited uptake of acetylated LDL.

hECFCs respond to changes in oxygen tension

Both hECFCs and HMVECs expressed high levels of HIF$1 \alpha$ when cultured at $1 \% \mathrm{O}_{2}$ similar to cells cultured in the presence of $\mathrm{CoCl}_{2}$ (Fig. 7). However, cells cultured at 5 or $21 \% \mathrm{O}_{2}$ revealed little to no HIF- $1 \alpha$ present in the cell lysates after $4 \mathrm{~h}$, suggesting that physiological oxygen levels are insufficient to induce HIF production. Similar results were observed with both cell types after $18 \mathrm{~h}$ (data not shown). 
Fig. 6 Acetylated LDL uptake by $\mathrm{hECFCs}(\mathbf{a}-\mathbf{c})$ and HMVECs (d-f) at 1,5 , and $21 \%$ oxygen. Images are representative of five independent experiments. Scale bars represent $200 \mu \mathrm{m}$
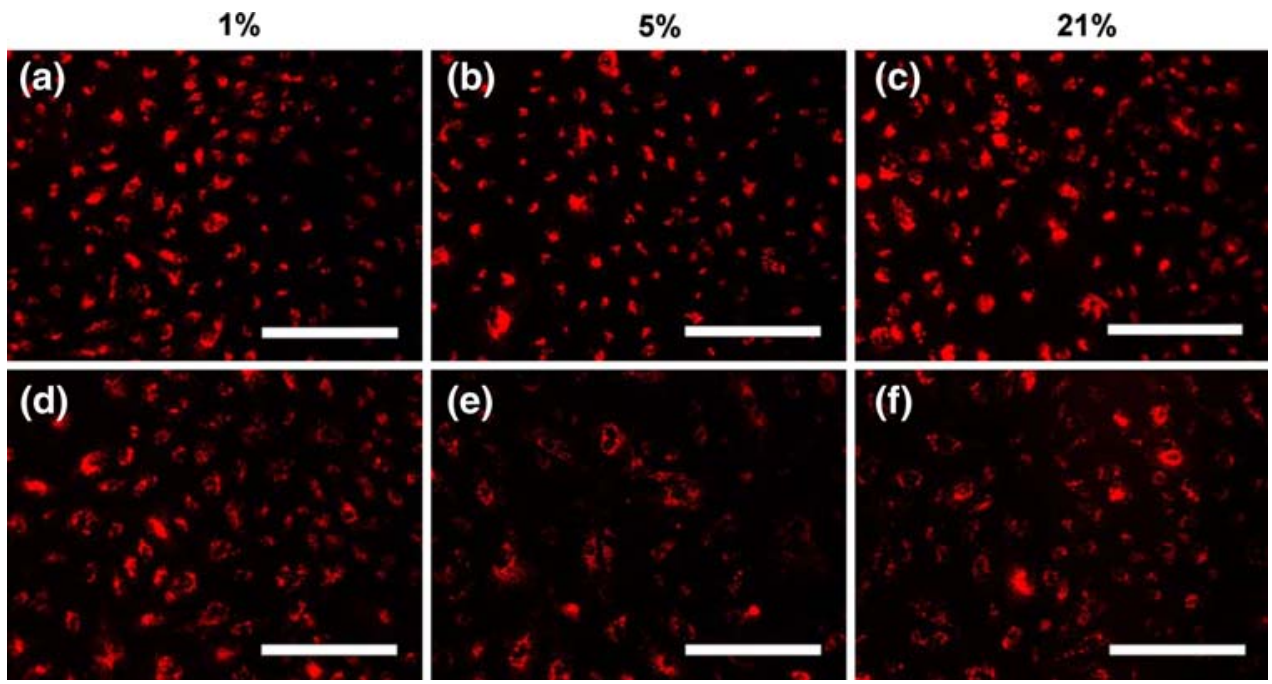

HIF-1 $\alpha$ $\alpha$-tub
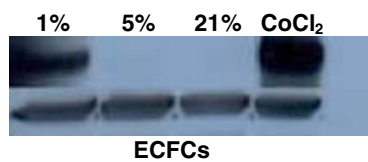
$1 \% \quad 5 \% \quad 21 \% \quad \mathrm{CoCl}_{2}$

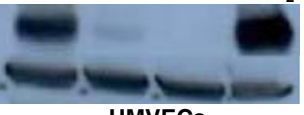
HMVECs

Fig. 7 Expression of HIF- $1 \alpha$ in hECFCs and HMVECs under varying oxygen tensions after 4 h. Bands represent protein expression at 1, 5, and $21 \%$ oxygen, or in the presence of $\mathrm{CoCl}_{2}$ (left to right). Lower band represents $\alpha$-tubulin expression

\section{Discussion}

Human endothelial colony-forming cells and HMVECs were studied using in vitro angiogenesis assays under varying oxygen tensions to test our hypothesis that hypoxic conditions could alter the proangiogenic potential of these cells. hECFCs performed as well or better than HMVECs when characterizing their proangiogenic potential, with particular emphasis on proliferation, migration, and tubulogenesis. These findings indicate that endothelial cell populations respond differently to hypoxic conditions and suggest that hECFCs may be a superior population for the neovascularization of ischemic or engineered tissues.

Endothelial colony-forming cells represent a highly proliferative population of circulating endothelial cells that have recently been distinguished from other EPC populations by their lack of hematopoietic surface markers and their ability to promote vasculogenesis [18, 30, 31]. Much like other progenitor cell populations, the number and function of circulating ECFCs diminishes with age and deteriorating health but increases after acute myocardial infarction. These data suggest a possible link between the presence of these cells in the circulation, cardiovascular health, and ongoing repair $[32,33]$. Compared to terminally differentiated endothelial cells, the ability to isolate these cells noninvasively from cord blood provides a distinct advantage over skin biopsy.

We observed a strong correlation between oxygen tension and three hallmarks of angiogenesis: cell proliferation, migration, and tubule formation. hECFCs exhibited a significantly greater proliferative capacity compared to HMVECs at low oxygen tensions in vitro $\left(\ll 1 \%\right.$ and $\left.4 \% \mathrm{O}_{2}\right)$, simulating conditions found within hypoxic tissues in vivo. The increased proliferative potential observed with hECFCs under hypoxic conditions suggests the potential for accelerating vessel formation. Furthermore, these data suggest that fewer cells would be required for transplant in the clinical setting to form functional vessels compared to existing methods using HMVECs. Our results also confirm the increased proliferative capacity of hECFCs compared to HMVECs in ambient oxygen typically associated with cell culture $\left(21 \% \mathrm{O}_{2}\right)$. This proliferative ability effectively reduces the time required to achieve sufficient cell numbers for transplantation to treat ischemic tissues or incorporate within a tissue-engineered scaffold. Finally, the proliferative capacity of hECFCs over a wide range of oxygen tensions differed from that of HMVECs. HMVEC proliferation peaked near physiological oxygen tensions observed in the arterial system [19]. In contrast, hECFC proliferation plateaued at $8 \% \mathrm{O}_{2}$ and remained constant at the higher levels assessed. These data suggest that changes in the oxygen microenvironment may have less of an effect on the proliferative capacity of hECFCs compared to HMVECs.

Human endothelial colony-forming cells also appeared to possess a higher level of sensitivity to a chemotactic gradient compared to HMVECs when quantitating cell migration at reduced oxygen tensions ( 1 and $\left.5 \% \mathrm{O}_{2}\right)$. Measurements of fluorescence intensity revealed increased hECFC migration compared to HMVECs in the presence of a chemotactic gradient. These data suggest that hECFCs 
may be capable of enhanced localization to sites of hypoxic stimuli in vivo when compared to HMVECs. Further, the reduced migration observed with hECFCs in the absence of a cytokine gradient suggests that hECFCs may remain at the transplant site to participate in capillary formation and avoid migration away from the desired tissue.

Regardless of oxygen tension, we observed robust tubule formation and uptake of acetylated LDL for both hECFCs and HMVECs. Both cell populations formed capillary-like structures at all oxygen levels tested. However, we detected distinct differences in tubule morphology between cell types at the same oxygen tension, with hECFCs forming smooth and more well-rounded tubules compared to HMVECs. Similar to reports by others, cell aggregation limited the accuracy of quantitation of cells participating in tubular structures [34]. Although it is widely accepted that tubulogenesis and LDL uptake are insufficient to characterize the endothelial lineage of different progenitor cell populations [30], these assays aid in assessing the capacity of an endothelial cell to maintain a functional phenotype when exposed to harmful environmental stimuli such as hypoxia. We have shown that hECFCs cultured in oxygen tensions representative of ischemia exhibit characteristic behavior similar to mature microvascular cells residing within a hypoxic environment.

Our findings suggest that hECFCs are less sensitive to apoptotic stimuli than HMVECs. While an approximately 3.5-fold increase in HMVEC caspase activity was observed at $1 \% \mathrm{O}_{2}$ compared to ambient conditions, hECFC caspase activity increased by only approximately twofold. While hECFCs did express higher baseline caspase levels than HMVECs when cultured under ambient conditions, both cell types expressed nearly identical caspase concentrations under conditions approximating those within hypoxic tissue. These data suggest that both cell types may have similar survivability when transplanted into ischemic sites in vivo. These data are in agreement with prior studies by Ingram et al. [35] that reported increased ECFC apoptosis upon the introduction of peroxide-mediated oxidative stress and simulation of vascular injury. It is likely that the culture of cells in reduced oxygen may initiate oxygenresponsive cues similar to those stimulated by small molecules, and the presence or magnitude of these cues directly impact apoptosis.

In order to probe the mechanism for differences in proangiogenic potential, we investigated the upregulation of HIF- $1 \alpha$ by each cell type in response to oxygen tension. Our data revealed a stark upregulation of this potent transcription factor in reduced oxygen $\left(1 \% \mathrm{O}_{2}\right)$ for both cells, while little to no expression was observed at higher oxygen tensions. These results differ from earlier studies that reported only minor shifts in HIF- $1 \alpha$ expression across conditions in EPCs cultured under similar oxygen levels [36]. HIF- $1 \alpha$ upregulation signals the increase in transcription of growth factors such as VEGF that may, in turn, enhance endothelial cell proliferation and migration. However, we failed to detect significant increases in VEGF secretion by hECFCs compared to HMVECs in 1,5 , or $21 \% \mathrm{O}_{2}$ using ELISA (data not shown). The transplant of EPCs genetically engineered to overexpress HIF- $1 \alpha$ has demonstrated increased proliferation, migration, and capacity to promote reperfusion in vivo [37]. Our observation of comparable HIF upregulation and VEGF secretion in both cell types stimulated by local oxygen conditions excludes the contribution of endogenous proangiogenic molecules as the sole mechanism for the increased proliferative capacity observed with hECFCs. Additional studies that focus on the molecular differences in cell division are warranted to explain these findings.

\section{Conclusions}

These data demonstrate that the proangiogenic response of cells of the endothelial lineage is highly dependent upon local oxygen tension and may differ in response to varying oxygen levels. Further investigation of the mechanism(s) responsible for these findings may reveal the optimal endothelial cell populations and conditions for the neovascularization of ischemic sites.

Acknowledgments These studies were supported by a pilot grant from the Center of Excellence in Translational Human Stem Cell Research (NIH grant \# P50-HL085036) and the NIH T32 Molecular Imaging Training Grant (NIH grant \# EB003827). The authors thank Damian Genetos, $\mathrm{PhD}$, for experimental assistance.

Open Access This article is distributed under the terms of the Creative Commons Attribution Noncommercial License which permits any noncommercial use, distribution, and reproduction in any medium, provided the original author(s) and source are credited.

\section{References}

1. Lloyd-Jones D et al (2009) Heart disease and stroke statistics2009 update. A report from the American heart association statistics committee and stroke statistics subcommittee. Circulation 119:e21-e181

2. Ennett AB, Kaigler D, Mooney DJ (2006) Temporally regulated delivery of VEGF in vitro and in vivo. J Biomed Mater Res A 79:176-184

3. Kong HJ, Kim ES, Huang YC, Mooney DJ (2008) Design of biodegradable hydrogel for the local and sustained delivery of angiogenic plasmid DNA. Pharm Res 25:1230-1238

4. Leu A, Leach JK (2008) Proangiogenic potential of a collagen/ bioactive glass substrate. Pharm Res 25:1222-1229

5. Ennett AB, Mooney DJ (2002) Tissue engineering strategies for in vivo neovascularisation. Expert Opin Biol Ther 2:805-818

6. Hoefer IE, Piek JJ, Pasterkamp G (2006) Pharmaceutical interventions to influence arteriogenesis: new concepts to treat ischemic heart disease. Curr Med Chem 13:979-987 
7. Peters MC, Polverini PJ, Mooney DJ (2002) Engineering vascular networks in porous polymer matrices. J Biomed Mater Res 60:668-678

8. Nor JE, Christensen J, Mooney DJ, Polverini PJ (1999) Vascular endothelial growth factor (VEGF)-mediated angiogenesis is associated with enhanced endothelial cell survival and induction of Bcl-2 expression. Am J Pathol 154:375-384

9. Nor JE et al (2001) Engineering and characterization of functional human microvessels in immunodeficient mice. Lab Invest $81: 453-463$

10. Richardson TP, Peters MC, Ennett AB, Mooney DJ (2001) Polymeric system for dual growth factor delivery. Nat Biotechnol 19:1029-1034

11. Koike $\mathrm{N}$ et al (2004) Tissue engineering: creation of long-lasting blood vessels. Nature 428:138-139

12. Shepherd BR, Jay SM, Saltzman WM et al (2009) Human aortic smooth muscle cells promote arteriole formation by coengrafted endothelial cells. Tissue Eng Part A 15:165-173

13. Ingram DA et al (2004) Identification of a novel hierarchy of endothelial progenitor cells using human peripheral and umbilical cord blood. Blood 104:2752-2760

14. Asahara T, Isner JM (2002) Endothelial progenitor cells for vascular regeneration. J Hematother Stem Cell Res 11:171-178

15. Kalka C et al (2000) Transplantation of ex vivo expanded endothelial progenitor cells for therapeutic neovascularization. Proc Natl Acad Sci USA 97:3422-3427

16. O'Neill TJ 4th, Wamhoff BR, Owens GK, Skalak TC (2005) Mobilization of bone marrow-derived cells enhances the angiogenic response to hypoxia without transdifferentiation into endothelial cells. Circ Res 97:1027-1035

17. Rehman J, Li J, Orschell CM, March KL (2003) Peripheral blood "endothelial progenitor cells" are derived from monocyte/macrophages and secrete angiogenic growth factors. Circulation 107:1164-1169

18. Yoder MC et al (2007) Redefining endothelial progenitor cells via clonal analysis and hematopoietic stem/progenitor cell principals. Blood 109:1801-1809

19. Vaupel P (1990) Oxygenation of human tumors. Strahlenther Onkol 166:377-386

20. Fujii $Y$ et al (1994) Effect of hypoxia on proliferation of microvascular endothelial cells derived from Mongolian gerbil brain. Neurol Med Chir (Tokyo) 34:799-802

21. Mikhaylova M et al (2008) Hypoxia increases breast cancer cellinduced lymphatic endothelial cell migration. Neoplasia 10:380 389

22. Kroon ME, Koolwijk P, van der Vecht B, van Hinsbergh VW (2001) Hypoxia in combination with FGF-2 induces tube formation by human microvascular endothelial cells in a fibrin matrix: involvement of at least two signal transduction pathways. J Cell Sci 114:825-833

23. Kourembanas S, Hannan RL, Faller DV (1990) Oxygen tension regulates the expression of the platelet-derived growth factor-B chain gene in human endothelial cells. J Clin Invest 86:670-674

24. Namiki A et al (1995) Hypoxia induces vascular endothelial growth factor in cultured human endothelial cells. J Biol Chem 270:31189-31195

25. Faller DV (1999) Endothelial cell responses to hypoxic stress. Clin Exp Pharmacol Physiol 26:74-84

26. Masiello LM, Fotos JS, Galileo DS, Karin NJ (2006) Lysophosphatidic acid induces chemotaxis in MC3T3-E1 osteoblastic cells. Bone 39:72-82

27. Sheffield JB, Graff D, Li HP (1987) A solid-phase method for the quantitation of protein in the presence of sodium dodecyl sulfate and other interfering substances. Anal Biochem 166:49-54

28. Broxmeyer HE et al (2006) Cord blood stem and progenitor cells. Methods Enzymol 419:439-473

29. Voyta JC, Via DP, Butterfield CE, Zetter BR (1984) Identification and isolation of endothelial cells based on their increased uptake of acetylated-low density lipoprotein. J Cell Biol 99:2034-2040

30. Hirschi KK, Ingram DA, Yoder MC (2008) Assessing identity, phenotype, and fate of endothelial progenitor cells. Arterioscler Thromb Vasc Biol 28:1584-1595

31. Sieveking DP, Buckle A, Celermajer DS, Ng MK (2008) Strikingly different angiogenic properties of endothelial progenitor cell subpopulations: insights from a novel human angiogenesis assay. J Am Coll Cardiol 51:660-668

32. Huang L et al (2007) Acute myocardial infarction in swine rapidly and selectively releases highly proliferative endothelial colony forming cells (ECFCs) into circulation. Cell Transplant 16:887-897

33. Ingram DA et al (2008) In vitro hyperglycemia or a diabetic intrauterine environment reduces neonatal endothelial colonyforming cell numbers and function. Diabetes 57:724-731

34. Donovan D, Brown NJ, Bishop ET, Lewis CE (2001) Comparison of three in vitro human 'angiogenesis' assays with capillaries formed in vivo. Angiogenesis 4:113-121

35. Ingram DA et al (2007) Clonogenic endothelial progenitor cells are sensitive to oxidative stress. Stem Cells 25:297-304

36. Nagano $M$ et al (2007) Identification of functional endothelial progenitor cells suitable for the treatment of ischemic tissue using human umbilical cord blood. Blood 110:151-160

37. Jiang $M$ et al (2008) Angiogenesis by transplantation of HIF-1 alpha modified EPCs into ischemic limbs. J Cell Biochem 103:321-334 